\title{
Laboratory Safety of Capsaicin Inhalation in Healthy Younger and Older Populations Potential Template for Inhalation Research
}

Stuart M Brooks*, Thomas Truncale and Andrew Sams

Colleges of Public Health and Medicine, University of South Florida, Tampa Florida, USA

*Corresponding author: Stuart M. Brooks, Colleges of Public Health and Medicine, University of South Florida, Tampa Florida, USA, E-mail: sbrooks@health.usf.edu

Received date: August 01, 2016; Accepted date: August 29, 2016; Published date: September 03, 2016

Copyright: ( 2016 Brooks SM, et al. This is an open-access article distributed under the terms of the Creative Commons Attribution License, which permits unrestricted use, distribution, and reproduction in any medium, provided the original author and source are credited.

\begin{abstract}
Introduction: There are inconsistencies as to the effects of age on the human cough reflex. The investigation speaks to the Federal Drug Agency's (FDA) trepidations for conducting inhalation experiments with non-approved medications/chemicals. The investigation addresses the accuracy of the mixing methodology and analysis of capsaicin stability during seven months of storage.
\end{abstract}

Methods: There is stringent safety monitoring while conducting 12 serial $(0.49-1000 \mathrm{uMol})$ single breath capsaicin inhalation challenge testing (CICT) on 20 younger and 20 older healthy research volunteers using pharmaceutical-grade capsaicin (i.e., Investigational New Drug protocol-IND 69,642). The research design and subject safety measures are recommended by the Food \& Drug Agency (FDA) and approved by the Institutional Review Board (IRB). Mixing of inhaled capsaicin solutions are by a Registered Pharmacist and concentration verifications are by high performance liquid chromatography (HPLC). Potency of stored capsaicin (i.e., refrigerated and shielded from UV light) is examined over 7 months.

Results: There are neither adverse reactions nor statistically significant difference in capsaicin cough parameters for older and younger volunteers at any dose of capsaicin. Physiologic monitoring by spirometry, impulse oscillometry, exhaled breath nitric oxide, electrocardiography, blood pressure, pulse and oxygen saturation measurements do not change at any dose. There are differences between the concentrations of capsaicin solutions mixed by a Registered Pharmacist and actual capsaicin determination by HPLC. The differences in capsaicin concentrations are $28.1 \%$ lower for 0.49 uMol compared to a $2.2 \%$ lesser concentration for 1000 uMol solution. During storage, capsaicin remains stable for 3 -months but substantially falls by six $(p<0.03)$ and seven $(p<0.004)$ months, especially for the lower concentrations.

Conclusion: The results of this investigation embrace the safety of serial Inhalations of dilute pharmacologic grade capsaicin aerosol among older and younger normal volunteers. Incorporating rigorous FDA recommendations, inclusion of IRB oversight and monitoring by spirometry, impulse oscillometry, exhaled breath nitric oxide, electrocardiography, blood pressure, pulse and oxygen saturation measurements document inhalation safety at any dilute capsaicin aerosol dose. However, there are lower capsaicin values determined by HPLC compared to expected capsaicin concentrations when mixed by a Registered Pharmacist or after six months of storage. The investigation champions the opinion that inhalation studies involving FDA non-approved drugs or chemicals/ medications can be safely conducted when following the appropriate safety procedures such as in this research protocol.

Keywords: Capsaicin; Cough; Cough reflex; Inhalation agents; Inhalation challenge; TRPV1 receptor

\section{Introduction}

The human cough reflex is one of the most important mechanisms in protecting the respiratory tract from aspiration of foreign substances, fluid, microbial agents or objects as well as clearing aspirated materials and endogenously produced mucus from the respiratory tract [1]. Early explorations recognize the molecular integrator through which capsaicin-induces cough by binding to the transient potential receptor vanilloid, $\operatorname{TPRV}_{1}[1,2]$. Capsaicin binding to the $\mathrm{TRPV}_{1}$ receptor propagates nervous impulse through nonmyelinated C-fibers initiating the human cough reflex. Customarily, serial inhalations of dilute capsaicin solutions are the principal method for researching the human cough reflex (i.e. capsaicin inhalation challenge test, CICT) [2-5].

The current investigation addresses FDA safety concerns by conducting capsaicin inhalation experiments on healthy old and young research volunteers using a pharmaceutical-grade capsaicin Investigational New Drug protocol (IND 69,642). The subject safety procedures are instituted and there is approval by the Institutional Review Board (IRB). The experimental protocol incorporates detailed human safety precautions. Inhaled capsaicin solutions are mixed by a Registered Pharmacist. Prepared doses are analyzed by high performance liquid chromatography (HPLC) before administration. Finally, the storage stability of capsaicin solutions over a seven month period, refrigerated at $4^{\circ} \mathrm{C}$ with protection against ultraviolet light is examined. 


\section{Methods}

\section{Human inhalation studies}

Capsaicin inhalation challenge test (CICT): Vials mixed by a Registered Pharmacist are removed from the refrigerator 30 minutes before testing so that the mixture warms to room temperature before use. Then, one $\mathrm{ml}$ of the first (lowest concentration of $0.49 \mathrm{uM}$ ) concentration is inserted into the nebulizer, using a sterile syringe, with subsequent employment of the CICT procedure as described by Dicpinigaitis et al. [6]. The methodology necessitates that the subject takes just a single breath of capsaicin aerosol starting from end-tidal exhalation (i.e., functional reserve capacity) to deep inspiration (i.e., total lung capacity). Delivery of Capsaicin aerosol is by a DeVilbiss nebulizer controlled by a dosimeter (KoKo DigiDoser Spirometer, nSpire Health Integrated Respiratory Information System, Longmont, CO 80501). The dosimeter contains an automated dosing devise incorporated into the spirometer along with a precise, softwarecontrolled aerosol dosing process. Dry compressed air is used to power the nebulizer, and the pressure regulator is set to $50 \mathrm{lb}$./in ${ }^{2}$. Flow meter accuracy is checked with a rotameter. An inspiratory flow regulator valve limits the inspiratory flow rate to 0.5 liters per second guaranteeing a consistent and reproducible inspiratory effort with each breath. The nebulizer output is determined to be $1.007 \mathrm{~mL} /$ minute and the duration of the aerosol delivery is 1.2 seconds; each breath delivers $0.02 \mathrm{~mL}$ capsaicin aerosol. Single breaths of capsaicin are delivered in ascending order, with normal saline solution randomly interspersed to increase challenge blindness, until two or more coughs $\left(\mathrm{C}_{2}\right)$ and five or more coughs $\left(\mathrm{C}_{5}\right)$ are reached over the next 15 seconds after the dose is delivered. Overall, reproducibility with this method is shown to be quite good, with $90-100 \%$ of challenges yielding $C_{2}$ and $C_{5}$ values within two doubling concentrations [6,7].

Subject inclusion criteria: Specific research volunteer inclusion criteria insure subject homogeneity and normalcy as shown in Table 1. The informed consent is signed before any study-related test or procedure is performed. After signing the informed consent, screening tests are accomplished to establish that a subject is "healthy" and meet the requirements of the study.

\begin{tabular}{|l|l|}
\hline $\mathbf{1}$ & Men of ages 18 and 30 (young) or $55-92$ (old) years old. \\
\hline $\mathbf{2}$ & Not current cigarette smoker. If an ex-smoker then no smoking $\leq 10$ years and consumption of $\leq 10$ pack years. \\
\hline $\mathbf{3}$ & Volunteers for the study are willing to sign informed consent. \\
\hline $\mathbf{4}$ & Absence of abnormal screening tests. \\
\hline a. & $\begin{array}{l}\text { Responses to the questionnaire denying current and prior respiratory diseases (including asthma, emphysema, chronic bronchitis, sinusitis and interstitial lung } \\
\text { d9sase) and no current respiratory complaints (e.g., cough, wheezing, shortness of breath, allergic rhinitis and sinusitis). Subjects must not be taking any } \\
\text { cardiac medications or admit to a physician-diagnosed cardiac or respiratory condition. }\end{array}$ \\
\hline b. & Shows "normal" spirometry with FEV $\&$ FVC $\geq 75 \%$ predicted and $F E V_{1} / F V C \geq 69 \%$ \\
\hline c. & Impedance oscillometry (IO) is within normal limits \\
\hline d. & There is a "negative" physical examination of the chest with absence of wheezing and/or crackles on auscultation of the chest. \\
\hline e. & Exhaled Nitric Oxide concentration is $\leq 35$ ppb for younger and $\leq 65$ ppb for older groups. \\
\hline &
\end{tabular}

Table 1: Inclusion Criteria

Spirometry: Spirometry measurements are performed according to American Thoracic Society specifications [8]. Predicted values are taken from Hankinson and co-workers studies [9]. Spirometry employs a KoKo spirometer using a pneumotachograph in order to provide flow-volume loops and volume-time graphics; and, there is multiple incentive graphics for patient coaching (nSpire Health Integrated Respiratory Information System; $\mathrm{KoKo}^{\circ}$ Pulmonary Function, Longmont, CO 80501). Spirometry is performed during screening and following each dose of capsaicin. Measurements include the forced vital capacity (FVC), forced expiratory volume during the first second of forced exhalation $\left(\mathrm{FEV}_{1}\right)$, and $\mathrm{FEV}_{1} / \mathrm{FVC} \%$. The best value of at least three maneuvers is expressed as a percentage of the predicted value.

Impulse oscillometry system (IOS): Measurement of airway resistance by impulse oscillometry (IO) employs the Jaeger MasterScreen IO (JAEGER, Inc. - Hochberg, Germany. The foundation of $\mathrm{IO}$ is based on the application of small pressure oscillation $\left(1 \mathrm{~cm} \mathrm{H}_{2} \mathrm{O}\right)$ at the mouth during spontaneous breathing and on the measurement of the respiratory resistance (respiratory impedance), at a frequency higher than the breathing rate $[10,11]$. The
IO method can differentiate between proximal $\left(R_{5}\right)$ and distal airways $\left(R_{20}\right)$ resistance as well as measures airway resistance at resonance frequency $\left(F_{R E S}\right)$. A significant change is deemed $\geq 10 \%$ increase in $R_{5}$ or $\mathrm{R}_{20}$. IO maintains an advantage over spirometry since multiple repeated maximal forced expiratory efforts are not required; frequent forced expiratory exhalation maneuvers can be difficult for older and very young subjects). Additionally, IOS holds value for bronchial challenge testing [10].

Exhaled breath Nitric oxide ( $\mathrm{E}_{\mathrm{NO}}$ ): Measurements of $\mathrm{E}_{\mathrm{NO}}$ are made during screening and at the end of the CICT. $\mathrm{E}_{\mathrm{NO}}$ use is to identify potentially unrecognized lung disease. Preliminary $\mathrm{E}_{\mathrm{NO}}$ concentration became a subject's inclusion criteria Table 1 . The on-line measurements of $\mathrm{E}_{\mathrm{NO}}$ is made using a chemiluminescence analyzer (Sievers, $\mathrm{NOA}^{\mathrm{m}}$ 280i; Boulder, CO), which is sensitive to $\mathrm{NO}$ concentration range of $\sim 0.5 \mathrm{ppb}$ to $500,000 \mathrm{ppb}$. Calibration involves generating NO-free calibration gas by passing ambient air through a NO scavenger device containing potassium permanganate $\left(\mathrm{KMnO}_{4}\right)$ and charcoal. Daily calibrations of the analyzer are completed prior to measurements using zero NO air and NO gas of 45 parts per million (ppm) (Sievers; Boulder, CO). Testing follows the guidelines of the American Thoracic 
Society [12]. The subject sits comfortably and has a mouthpiece adjusted to the proper height and position without use of nose clips. After tidal breathing, the subject inhales to total lung capacity and then exhales at a flow rate of $0.05 \mathrm{~L} /$ second against a fixed expiratory resistance. This technique insures velum closure and eliminates possible contamination with upper airways nitric oxide. There is achievement of a constant flow rate through biofeedback by showing a target flow rate on a computer display. With this biofeedback approach, all subjects are able to maintain the desired expiratory flow rate. The duration of exhalation continues for at least six seconds until there is achievement of a three-second plateau. There is repetition of exhalations in order to obtain at least two NO plateau values that agrees within $10 \%$ of each other and the exhaled NO concentration is calculated as the mean of two values.

Symptom questionnaire: There is incorporation of a Symptom Questionnaire (SQ) at baseline and again after each capsaicin dose; SQ is also administered at one and five days after the CICT. The questionnaire is a modification of one used by Ternesten-Hasseus [13]. For each category of symptom, there is an intensity value based on a four-item Likert scale: (4) Very much; (3) moderate amount; (2) very little; (1) none. Therefore, the highest score on the "amount" scale is " 4 "; the lowest score representing no exposure (none) is " 1 ". The questionnaire asks, "At this point in time, to what degree do you note the following symptoms?" (1) Heaviness or difficulty in your breathing; (2) mucous production: (3) runny or irritated nose or nasal passages; (4) throat irritation or burning sensation: (5) sensation of a "weight" or tightness of the chest; and, (6) feeling of chest burning.

Monitoring of subjects during CICT: SQ is completed after each inhaled capsaicin dose. Monitoring of the subjects' responses throughout the CICT includes gaging pulse rate and blood pressure, noting electrocardiogram tracing and assessing percentage oxygen saturation. There is the determination of $\mathrm{E}_{\mathrm{NO}}$ at baseline and after the final capsaicin dose. A follow-up telephone survey administers the SQ at 24 ( 1 day) hours and 120 hours (5 days) following the CICT. During each telephone call, the subject is asked the 6 questions from the SQ and the results are compared to the baseline and post-CICT responses. Any subject reporting persistent symptoms of category \#4 (i.e., very severe) for two or more items, compared to the baseline, is asked to return to for further evaluation. There is examination of the nose and throat and auscultation of the chest. A spirometry tracing will be obtained. IO $\mathrm{E}_{\mathrm{NO}}$ measurements are obtained.

Adverse events: (serious and / or "unexpected adverse event"): Subject showing one or more of the following post-capsaicin challenge manifestation (at any capsaicin dose) is judged to display an "unexpected adverse effect:" (1) persistent wheezing on auscultation of the chest; (2) $\geq 12 \%$ fall in FEV1 or FVC compared to baseline; (3) respiratory resistance (by IO) of $\geq 4$ times the baseline value; (4) continuing complaint of \#4 severity for at least 2 items of the SQ; (5) persistent elevated exhaled ENO $\geq 2 \frac{1}{2}$ times baseline value. Subjects deemed to have suffered an "unexpected adverse event" will undergo one week of treatment with an inhaled corticosteroid and aerosol bronchodilator. After 1 week of treatment, subjects would be reevaluated in a similar manner as above.

Reason to terminate study: Termination of the study will occur if at least five (5) subjects suffer an "unexpected adverse event". The 5 value represents $12.5 \%$ of a study population of 40 persons.
Emergency response: The investigators are trained in life-saving procedures. Emergency response equipment is present in the laboratory at the time of testing.

\section{Capsaicin Preparation and Measurement}

\section{Pharmaceutical grade capsaicin}

Purchase of pharmaceutical grade capsaicin, acceptable to an FDA protocol, derives from Formosa Laboratories Inc. and stored at $-20^{\circ} \mathrm{C}$ until use. (Formosa Laboratories, Inc. Taoyuan, Taiwan 338. Lot Number F0010201; URL: http://www.formosalab.com

\section{High performance liquid chromatography (HPLC)}

Administered capsaicin doses are analyzed by high performance liquid chromatography (HPLC) using a Varian HPLC Star \# 1 (ProStar/Dynamax Systems equipped with Solvent delivery Module 210, ProStar injector/controller; ProStar Fluorescence 363, and Autosampler 410). The mobile phase is 75\% Methanol; 25\% HPLC grade water. The columns are Inertsil 5u ODS-3 $250 \times 4.6 \mathrm{~mm}$, Part \# A 0396-250x046 Lot \# TQ5- 2107, Serial \# 4JI86141; column temperature is $28^{\circ} \mathrm{C}$. Detector: Fluorescence: Excitation $281 \mathrm{~nm}$; Emission $312 \mathrm{~nm}$; flow rate of $1.2 \mathrm{I} /$ minute for ten minutes. HPLC is set according to manufacture recommendations.

\section{Mixing capsaicin solutions}

A $\mathrm{PhD}$ registered pharmacist mixes the pharmaceutical grade capsaicin stock solution with individual capsaicin doses provided via sterile conditions using a hood with exhaust ventilation. Powdered capsaicin (8-methyl-n-vanillyl-6-nonenamide) (30.5 g) is mixed with $10 \mathrm{ml} 100 \%$ ethyl alcohol (USP), $10 \mathrm{ml}$ Tween 80 and $80 \mathrm{~mL} 0.9 \%$ saline to constitute the stock solution with a final capsaicin concentration of $3.05 \mathrm{mg} / \mathrm{ml}$ or $0.01 \mathrm{Mol} /$ liter. Subsequently, the stock solution is further diluted with physiologic saline solution to yield 12 serial doubling concentrations from 0.49 to $1,000 \mathrm{uMol} / \mathrm{L}$. The final 12 diluted capsaicin concentrations are: $0.49,0.98,1.95,3.9,7.8,15.6$, $31.2,62.5,125,250,500$, and $1000 \mathrm{uMol} / \mathrm{L}$. Stock solutions and individual doses are first passed through a Millipore filter (steam sterile filter of 0.25 micron) to insure sterility. Random sample from each batch are also sent to a laboratory for culture. Each capsaicin dose is placed in an individual sterile vial and stored in a refrigerator, shielded from ultraviolet light at $4^{\circ} \mathrm{C}$. The final series of mixed samples were organized as 40 sets of vials with each set containing 12 concentrations of Capsaicin mixed by the pharmacist over of the necessary range of concentrations $(0.49-1000 \mathrm{uMol} / \mathrm{L})$. All of the sets of vials were then transferred to the laboratory where the CICTs were conducted.

\section{Capsaicin quality control}

After the sets of capsaicin solution vials are received form the pharmacist, further investigations are accomplished by HPLC analyses. Only pharmaceutical grade capsaicin is used for the human inhalation studies. Assurance of the accuracy of HPLC analyses of capsaicin solutions is accomplished by pre-calibrating the HPLC using fresh pharmaceutical grade capsaicin solutions. For the HPLC analyses, a stock solution is dispensed by dissolving $12.5 \mathrm{mg}$ of pharmaceutical grade capsaicin in acetonitrile and diluting to $25 \mathrm{~mL}$. The calibration standards were constructed by mixing a minimum of five different concentrations in acetonitrile. Pharmaceutical grade capsaicin stock 
Page 4 of 8

solution for HPLC is $0.5 \mathrm{mg} / \mathrm{mL}(500 \mathrm{ug} / \mathrm{mL})$ and there are five calibration working standards of $0.1,0.5,1.0,3.0$, and $6.0 \mathrm{ug} / \mathrm{mL}$. Recovery of the Initial freshly mixed pharmaceutical grade calibration standard is found to be between $95 \%$ and $105 \%$ of the calculated value. To prepare the calibration standards, solutions are mixed in a $10 \mathrm{ml}$ volumetric flask. The appropriate amount of capsaicin is added into the volumetric flask, which is filled to the bottom of the neck with acetonitrile. The volumetric flask is then brought up to volume and inverted five to ten times to insure that the standard is properly mixed. The calibration standard is then transferred to a vial with Teflon-lined screw cap for storage. Pharmaceutical grades capsaicin of ten microliter (uL) sample aliquots is injected into the HPLC via the autosampler. When the peak area is above the range of the working standards, it is diluted, reanalyzed and appropriate dilution factor are used in calculations. Linearity of calibration curve is obtained with $\mathrm{r}^{2}=0.9999$. Before stored capsaicin solutions (obtained from the pharmacist) are analyzed, the calibration curve is verified with an initial calibration verification check standard (ICV) to see if the calibration curve is holding. There are essentially identical correlations when the solutions are re-tested during the duration of the study (3months). Reference standard are between $95 \%$ and $105 \%$ of the calculated value. In order to test the adequacy of the pharmaceutical grade capsaicin, we performed identical studies using a second source of capsaicin that has been utilized by other investigators [6,7]. This second check capsaicin standard (CCV) standard is purchased from Sigma-Aldrich, Saint Louise, MO 63103 (Catalog Number 12084 Brand: FLUKA). Recovery of the CCV is between $90 \%$ and $110 \%$ of the pharmaceutical grade capsaicin value. The CCV is verified after each ten field samples and at the end of the analytical sequence. When any CCV falls outside the acceptable limit then all samples and QC sample are rerun. Stock solution is stored, covered with aluminum foil, at $4^{\circ} \mathrm{C}$ for seven months.

\section{Capsaicin storage}

There are 16 sets of capsaicin solutions received from the pharmacist, made up of different concentrations (0.49-1000 uMol), stored at $4^{\circ} \mathrm{C}$ and covered with aluminum foil to protect against ultraviolet light for up to 7 months. Periodic HPLC analyses of the samples are made over this time period. The capsaicin calibrating solutions are analyzed before measurements of the stored solutions using fresh pharmaceutical grade capsaicin solutions of the five working standards. Control samples are analyzed with each run of the calibrator and patient samples. In general, there is excellent reproducibility with all repeated analyses.

\section{Statistical analysis}

The calibration data is analyzed statistically with means, standard errors of the mean and standard deviations. Student T-test is utilized to test differences between doses. The frequencies of the demographic characteristics and the median ages are determined for the young and the old groups. From the spirometric data, median $\mathrm{FEV}_{1}$ (percentage of normal), median $\mathrm{FEV}_{6}$ (percentage of normal), and median $\mathrm{FEV}_{1} /$ $\mathrm{FEV}_{6}$ are concluded. The $\mathrm{E}_{\mathrm{NO}}$ values are compared for the younger and older groups using the Wilcoxon Rank Sum test. Application of the Holm method is applied to limit the chances of Type I error introduced due to multiple testing.

\section{Results}

\section{Study population}

Table 1 conveys the Inclusion Criteria for admission to the study while Table 2 displays Baseline Value for various tests on 20 younger and 20 older male subjects. The baseline $\mathrm{FEV}_{1}$ is higher for the younger group $(\mathrm{p}<0.01)$ but all values are within normal predicted values for age. Older subjects show higher baseline $\mathrm{E}_{\mathrm{NO}}(\mathrm{p}<0.006)$, which is consistent with previous findings [14]. There is no significant change in $\mathrm{E}_{\mathrm{NO}}$ after completion of the CICT.

\begin{tabular}{|c|c|c|}
\hline Measurement & Young $(\mathrm{N}=20)$ & Old $(\mathrm{N}=20)$ \\
\hline Age, years,(Mean $\pm S D)$ & $21.8 \pm 2.4$ & $68.3 \pm 7.81$ \\
\hline $\mathrm{E}_{\mathrm{NO}}, \mathrm{ppb}(\text { Mean } \pm \mathrm{SD})^{*}$ & $19.8 \pm 8.0$ & $35.0 \pm 14.5^{*}$ \\
\hline Total Coughs (Mean \pm SD) & $12.0 \pm 5.0$ & $11.5 \pm 5.1$ \\
\hline Mean $\mathrm{C}_{2}$, uMol $($ Mean $\pm \mathrm{SD})$ & $21.2 \pm 11.3$ & $23.4 \pm 12.1$ \\
\hline Mean $\mathrm{C}_{5}$, uMol $($ Mean $\pm \mathrm{SD})$ & $90.6 \pm 48.2$ & $67.7 \pm 27.1$ \\
\hline$F_{\text {res }}$, L.s. $^{1}($ Mean $\pm S D)$ & $11.2 \pm 3.1$ & $15.5 \pm 3.6$ \\
\hline$R_{5}$, L.s- $^{-1}$ & $3.4 \pm 1.0$ & $3.7 \pm 1.0$ \\
\hline $\mathrm{R}_{20}$, L.s.-1 & $3.1 \pm 0.8$ & $2.9 \pm 0.64$ \\
\hline $\mathrm{FEV}_{1}, \mathrm{~L}$ & $4.4 \pm 0.6$ & $3.1 \pm 0.5^{* *}$ \\
\hline Symptom Quest. Score & $6.3 \pm 0.5$ & $6.3 \pm 0.4$ \\
\hline
\end{tabular}

Table 2: Mean values of two populations. ${ }^{*} \mathrm{p}<0.006,{ }^{* *} \mathrm{p}<0.01$.

\section{Capsaicin challenge testing}

There are no statistically significant differences in total coughs and $\mathrm{C}_{2}$ between young and older subjects Table 2 although older subjects show a higher mean $C_{5}$ than younger subjects $(90.6 \pm 48.2$ vs. $67.7 \pm$ 27.1). As shown in Figure 1, there are no significant differences in total coughs, $\mathrm{C}_{2}$ and $\mathrm{C}_{5}$ between the two groups. There are no occurrences of adverse symptoms or physiological responses in any subject at any capsaicin dose. No subject requires emergency treatment and no subject needs termination from the study because of persistent responses. Two subjects in the younger group and no subjects in the older group do not reach $\mathrm{C}_{5}$ even at the highest dose of $1000 \mathrm{uMol}$. These latter two subjects experience no adverse symptoms or physiologic changes. There are no significant parameter changes during continuous measurement of pulse rate, blood pressure, electrocardiogram tracing and oxygen saturation. Auscultation of the chest is negative for wheezing and/or crackles in all subjects after every capsaicin dose. There are no statistically significant changes in $\mathrm{FEV}_{1}$ for any subject at all doses as shown in Figure 2. However, $\mathrm{FEV}_{1}$ values are uniformly higher for younger subjects as expected. Serial testing with IO values $\left(R_{5}, R_{20}, F_{\text {res }}\right)$ and SQ scores reveal no significant changes Figure 3.

\section{Delayed response}

No subject develops a delay of symptoms by follow-up telephone confirmation 24 hours (1-day) and 120 hours (5-days) following the CICT challenge. 


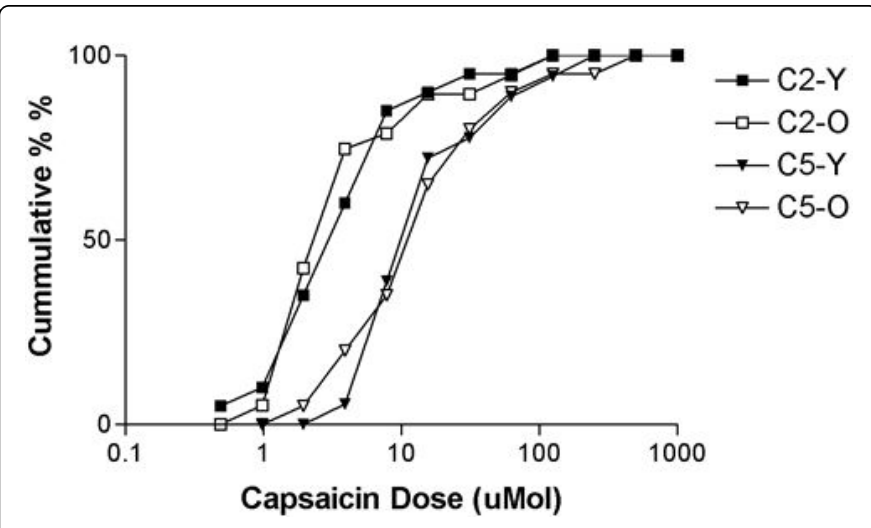

Figure 1: Comparison of Capsaicin dose-responses between younger $(\mathrm{Y})$ and older $(\mathrm{O})$ subjects. There is no significant difference between groups.

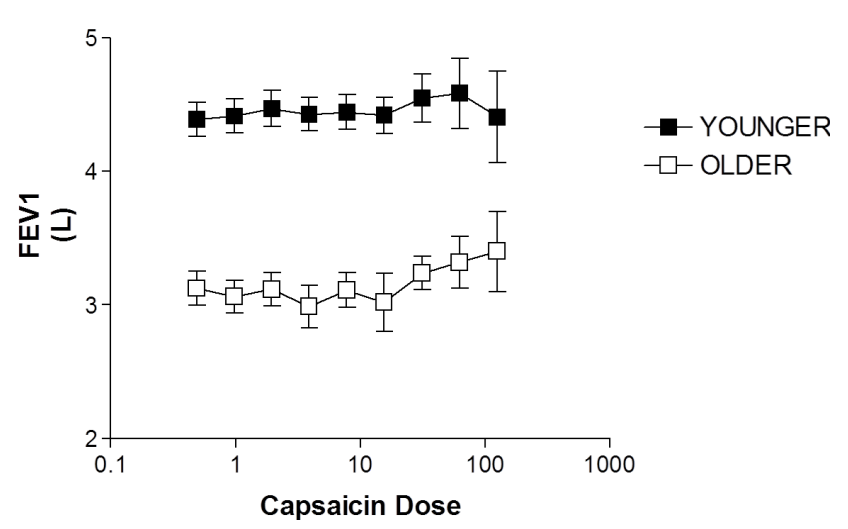

Figure 2: Comparison of $\mathrm{FEV}_{1}$ for young and old subjects at different capsaicin doses. There were no significant changes in $\mathrm{FEV}_{1}$ at any capsaicin dose including the highest capsaicin concentration (uMol).

\section{Capsaicin analyses}

Table 3 displays mean, standard deviation, range, $\%$ predicted (mixed $\div$ actual concentrations $\mathrm{x} 100$ ) and coefficient of variation. Analysis of the $1000 \mathrm{uMol} / \mathrm{L}$ capsaicin stock solution reveals a difference between the value mixed by the Registered Pharmacist and the determination of capsaicin concentration by HPLC. Only $97.4 \%$ of the $1000 \mathrm{uMol} \div \mathrm{L}$ capsaicin is dissolved in solution resulting in an HPLC-determined concentration of $976.8 \mathrm{uMol} / \mathrm{L}$ (2.6\% reduction). Additionally, the serial dilutions of 12 concentrations of the stock solution (administered to 36 subjects) also shows differences comparing the concentrations by HPLC to assumed/predicted dose of capsaicin after mixing by the Registered Pharmacist. The mean percentage HPLC measurement $\div$ pharmacist calculation is $85.5 \%$. Differences are greatest for the lower concentrations in comparison to the higher capsaicin dilutions. The percentage predicted ranges between $71.9 \%$ for the lowest concentration of $0.49 \mathrm{uMol}$ and $97.8 \%$ for the $1000 \mathrm{uMol}$ concentration. The difference is $28.1 \%$ less than expected for the $0.49 \mathrm{uMol}$ concentration in comparison to a $2.2 \%$ lesser concentration for the $1000 \mathrm{uMol}$ solution of capsaicin. There are statistically significant differences for the first ten solutions (concentrations $0.49-250 \mathrm{uMol}, \mathrm{p}<0.03$ ) while there is no statistically significant differences for the last two concentrations (500 and 1000 $\mathrm{uMol})$. The $\%$ coefficient of variation $(\% \mathrm{CV})$ or relative standard deviation (\% RSD) is a dimensionless number allowing comparison of the variation of measurements variation having significantly different mean values. The \% CV demonstrates small differences except at the lowest concentration. The \% CV is greatest (e.g., 5.42\%) at the 0.49 $\mathrm{uMol} / \mathrm{L}$ concentration and lowest (e.g., 2.01\%) at the concentration of $1000 \mathrm{uMol} / \mathrm{L}$.

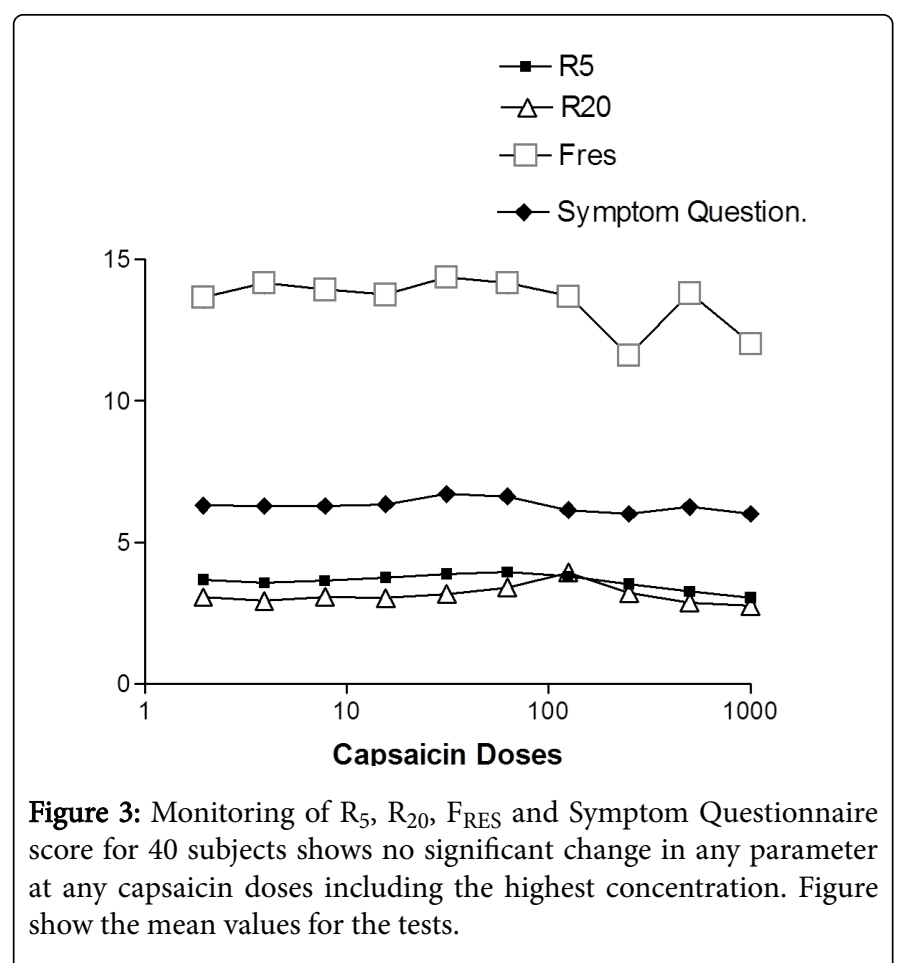

\section{Capsaicin storage}

For the first 3-months of storage, the capsaicin concentrations are relatively stable with no statistically significant difference in comparison to baseline (Table 4). The concentrations average 15\% less than dose assumed by the Registered Pharmacist after mixing. There is greater capsaicin concentration reduction at six $(\mathrm{p}<0.03)$ and seven $(\mathrm{p}<0.004)$ months. At 7 months, the lowest concentration of $0.49 \mathrm{uMol}$ decreases to $44.9 \%$ of predicted (a $14.3 \%$ fall from baseline). In contrast the higher concentrations appears to remain relatively stable for at least 7 months.

\section{Discussion}

Several publications proclaim the clinical safety of CICT [15-18]. A rationale for the current investigation, focusing on age, is because there are still inconsistencies concerning the role of age on the human cough reflex $[19,20]$. The present study accomplishes CICT on normal younger and older volunteers employing strategies heightening volunteer safety [21]. First, there is the submission and acceptance of the research protocol by the Food and Drug Agency (FDA) and assurance by the Institutional Review Board (IRB). The investigation employs a more than $99 \%$ pure pharmaceutical grade capsaicin via an Investigational New Drug (IND 69,642) submission [21]. A Registered 
Page 6 of 8

Pharmacist mixes individual pharmaceutical grade capsaicin doses because purity and solubility are essential qualities for the capsaicin stock solution and are prerequisites for accurate dilute capsaicin doses [5,22-24]. Analyses of prepared capsaicin solutions are by high performance liquid chromatography (HPLC); other investigators employ ultraviolet absorption spectrophotometry to determine capsaicin concentrations [22]. Volunteers sign a consent form mentioning unfavorable study risks. During individual serial monitoring of the CICT, there is integration of the symptom questionnaire, spirometry, impulse oscillometry, exhaled breath Nitric Oxide, electrocardiography, blood pressure levels, pulse rate and percentage oxygen saturation. None of the afore mentioned parameters change during CICT.

\begin{tabular}{|l|l|l|l|l|}
\hline Predicted & Mean \pm SD & Range & \%Predicted & \%CV \\
\hline 0.49 & $0.35 \pm 0.019$ & $0.32-0.41$ & 71.9 & 5.42 \\
\hline 0.98 & $0.78 \pm 0.025$ & $0.74-0.83$ & 79.2 & 3.17 \\
\hline 1.95 & $1.64 \pm 0.043$ & $1.56-1.71$ & 84.1 & 2.63 \\
\hline 3.9 & $3.17 \pm 0.109$ & $2.96-3.46$ & 81.4 & 3.4 \\
\hline 7.8 & $6.34 \pm 0.190$ & $6.06-6.82$ & 81.3 & 3 \\
\hline 15.6 & $12.78 \pm 0.35$ & $12.16-13.59$ & 81.8 & 2.76 \\
\hline 31.2 & $26.34 \pm 0.67$ & $24.79-27.92$ & 84.4 & 2.56 \\
\hline 62.5 & $54.55 \pm 1.67$ & $51.61-57.99$ & 87.3 & 3.07 \\
\hline 125 & $111.40 \pm 1.94$ & $108.42-115.50$ & 89.1 & 1.74 \\
\hline 250 & $228.85 \pm 5.80$ & $221.28-239.47$ & 91.5 & 2.53 \\
\hline 500 & $471.24 \pm 11.09$ & $452.46-504.10$ & 94.2 & 2.35 \\
\hline 1000 & $977.52 \pm 19.71$ & $943.83-1012.48$ & 97.8 & 2.01 \\
\hline
\end{tabular}

Table 3: Capsaicin analyses comparing predicted value with actual amount of capsaicin as measured by Hplc. Values are reported in $\mathrm{uMol} / \mathrm{L} ; \%$ Predicted $=\%$ predicted; $\% \mathrm{CV}=\%$ Coefficient of Variation.
Importantly, the investigation identifies issues needing further study. There is scrutinizing of dissimilarities between the concentrations of capsaicin solutions mixed by a Registered Pharmacist and the different determinations of capsaicin concentrations by HPLC measurements. The differences are principally for the lower concentrations in comparison to the higher capsaicin dilutions. These findings are in contrast to the studies reported by Kopec et al. Who observe lower concentrations of capsaicin to be relatively stable while higher concentrations display lesser values than predicted [23].

The precise capsaicin dosing concentrations for conducting the CICT is not fully established. Our dosing methodology, without adverse effects, involves aerosolizing single breath concentrations of capsaicin, ranging between $0.49 \mathrm{uMol}$ to $1000 \mathrm{uMol}$ as endorsed by Dicpinigaitis [6,7]. Vovk et al. determine that aerosolizing $200 \mathrm{uMol}$ capsaicin is a suprathreshold concentration for eliciting the $\mathrm{C}_{5}$ response in healthy participants [25].

Some investigators employ a much higher capsaicin dosing schedule than in use by the current researchers $[13,26]$. The investigators reduce the amount of aerosolized capsaicin in subsequent studies [27,28]. In the 2002 investigation, the protocol entails inhaling capsaicin aerosol, by tidal ventilation, for a total of 6 minutes to induce coughing followed by 4 minutes of rest [13]. The numbers of capsaicin-evoked coughs are counted for the entire 10 minutes from the onset of provocation [13]. When more than 70 coughs develop from a specific dose of capsaicin, provocation is terminated. The administration of specific concentrations include $0.40,2.0$ and $10 \mathrm{uMol} / \mathrm{L}$. Typically, coughing is most intense during the first minutes of the challenge. Nineteen $(50 \%)$ patients react with severe coughing ( $>70$ coughs) after the 6-minute inhalation of the first dose of $0.4 \mathrm{uMol} / \mathrm{L}$. There is provocation of five patients ( $>70$ coughs) after the second dose of capsaicin $(2.0 \mathrm{uMol} / \mathrm{L})$, and provocation occurs in 14 volunteers after the highest dose $(10 \mathrm{uMol} / \mathrm{L})$. In contrast, all normal (i.e., control subjects without disease) are able to inhale all three levels of capsaicin without developing more than 70 coughs.

\begin{tabular}{|l|l|l|l|l|l|l|l|l|l|l|l|l|}
\hline $\mathbf{M}$ & 0.49 & 0.98 & 1.98 & 3.9 & 7.8 & 15.6 & 31.2 & 62.5 & 125 & 250 & 500 & 1000 \\
\hline $\mathbf{0}$ & 0.34 & 0.77 & 1.67 & 3.38 & 3.38 & 13.27 & 27.26 & 54.66 & 111.11 & 226.96 & 473.11 & 975.9 \\
\hline $\mathbf{1}$ & 0.34 & 0.76 & 1.64 & 3.3 & 3.3 & 12.8 & 26.49 & 55.07 & 111.6 & 230.1 & 479 & 983.8 \\
\hline $\mathbf{2}$ & 0.34 & 0.76 & 1.62 & 3.17 & 3.17 & 12.73 & 26.12 & 54.37 & 111.8 & 228 & 468.4 & 977.2 \\
\hline $\mathbf{3}$ & 0.36 & 0.8 & 1.65 & 3.12 & 3.12 & 12.64 & 26.21 & 54.73 & 111 & 229.6 & 467.9 & 975.1 \\
\hline $\mathbf{7}$ & 0.29 & 0.66 & 1.35 & 2.51 & 2.51 & 11.16 & 23.01 & 51.5 & 106.9 & 222.2 & 473.4 & 980.2 \\
\hline $\mathbf{8}$ & 0.27 & 0.56 & 1.3 & 2.46 & 2.46 & 10.69 & 22.44 & 48.34 & 100.9 & 210.2 & 459.3 & 957.2 \\
\hline
\end{tabular}

Table 4: Capsaicin solutions stored in a refrigerator and shielded from ultraviolet light. $(\mathrm{M}=$ month of storage); Values obtained by HPLC analyses. Original concentrations determined by registered pharmacist are reported in uMol/L (upper column). There is no significant difference for measurements at month 1 and 3 of storage. By month 6 and 7, substantial reductions are noted with reduction greatest for lower capsaicin concentrations.

If we make a dose-by-dose comparison of the Ternesten-Hasseus study with our own testing, we recognize a difference [13]. The comparison of a $2.0 \mathrm{uMol} / \mathrm{L}$ dose (breathing continuously for 6 minutes) with our $2 \mathrm{uMol}$ dose (1 breath) will deliver roughly 1/500 capsaicin. Furthermore, our endpoint of 5 coughs vs. 70 coughs is approximately $1 / 14$ less cough outcome. Our more safe testing procedure requires a single breath per dose and the dosing began at an 
extremely low concentration; there is an endpoint of 5 coughs with concentrations ranging between 0.49 and $1,000 \mathrm{uMol} / \mathrm{L}$.

We cannot explain why our capsaicin solutions become unstable after 6 months while the study of Kopec et al. report capsaicin stability lasting for 1 year [23]. Kopec's study values are the opposite of ours; the lower concentrations of capsaicin are relatively stable while the higher capsaicin concentrations display lower values than the predicted. Perhaps, the reason for the departure is because solutions containing more capsaicin tend to aggregate into a more stable complex. Kopec et al. hypothesize that the discrepancies in capsaicin measurements may be due to the mixing with $100 \%$ ethyl alcohol and $0.9 \%$ saline, which makes the solution unstable. Moreover, more saline is used as dilution progress and perhaps causes capsaicin precipitation out of solution. More dilution with saline means relatively less ethyl alcohol and Tween 80 , a requirement for dissolving capsaicin and leading to capsaicin precipitation out of solution.

Our investigative protocol, attempting accurate measurements of concentration mixtures, better verifying chemical purity and solubility and inclusion of strict provisions ensuring volunteer safety may act as a template for other inhalation investigations. Likely, future investigations will embrace protocols incorporating the inhalation of different medications and/or industrial chemicals [29-33]. Scrutiny is necessary to insure human safety as more and more medications and chemicals are being administered by the inhalation route, including drugs and chemicals produced as nanoparticles [31-33]. Investigations will be driven by innovative technology and novel research concepts while some studies will involve research dealing with ethical issues [34]. Not all research protocols are equally ethical and there is a need to provide appropriate protection for all research participants [35].

An imperative human safety deliberation is for specific inhalation challenge (SIC) testing using occupational agents, such as toluene diisocyanates or organic dusts. This practice, while readily available in Canada and Europe, is limited in the United States. Purportedly, human inhalational studies using occupational agents do not require FDA approval or protocols such as used in this investigation. However, the FDA and IRBs are becoming more rigorous in their requirements for subject safety and more stringent protocols will be required in the future. We suggest that the investigative approach of the current investigation can apply to drug/chemical inhalation studies where subject safety is in question Table 5 . We believe that the use of occupational agents to perform specific inhalation challenges can be conducted safely when inhalation testing follows the appropriate safety procedures such as in this investigation or perhaps investigations espoused by others [36].

\begin{tabular}{|l|l|}
\hline $\mathbf{1}$ & Document purity of raw chemical/drug by use of pharmaceutical grade or other criteria \\
\hline $\mathbf{2}$ & May require FDA IND application \\
\hline $\mathbf{3}$ & Approval by institutional review board (IRB) \\
\hline $\mathbf{4}$ & Include mixing of chemical/drug by registered pharmacist before use \\
\hline $\mathbf{5}$ & Document purity and sterility of prepared chemical/drug mixture by pharmacist (e.g., HPLC, other approaches) \\
\hline $\mathbf{6}$ & Accurate documentation of dosage administered to subject (HPLC, etc.) \\
\hline $\mathbf{7}$ & Strict inclusion and exclusion criteria of tested subjects \\
\hline $\mathbf{8}$ & Accurate description of risks in the consent form \\
\hline $\mathbf{9}$ & Serial monitoring using lung function testing. \\
\hline $\mathbf{1 0}$ & $\begin{array}{l}\text { Inclusion of monitoring for systemic responses (BP, pulse rate, } \mathrm{O}_{2} \text { saturation, etc.) during dosing or inhalation and, if appropriate, use other indicators of lung } \\
\text { injury (exhaled breath parameters, induced sputum, blood tests, etc.) }\end{array}$ \\
\hline $\mathbf{1 1}$ & Monitor for delayed effects \\
\hline
\end{tabular}

Table 5: Guidelines for human research involving drugs/chemicals not approved for inhalation use.

\section{Conclusions}

The human cough reflex is one of the most important mechanisms in protecting the respiratory tract from aspiration of foreign substances, fluid, microbial agents or objects. In addition, it is the primary means of clearing aspirated materials and endogenously produced mucus from the respiratory tract.

Our data holds several implications for the performance of capsaicin challenges. The findings of the current investigation suggest other studies likely have overestimated the actual concentrations of their capsaicin solutions, thus calling into question both the accuracy and the reproducibility of the data. There is the need to insure human subject safety during research investigations employing inhalation of medications or chemicals fostered by new technological approaches or novel research concepts [31-33]. Such investigations can be safely conducted when they follow the appropriate safety procedures such as described in this investigation Table 5. Specifically, for investigators performing CICT, inhalation of dilute capsaicin aerosols is relatively safe. The capsaicin inhalation effect is innocuous and short lived.

\section{Acknowledgement}

Partial funding for the investigation was derived from: $\mathrm{CDC}$ NIOSH T42-OH008438.

\section{References}

1. Brooks SM (2011) Perspective on the human cough reflex. Cough 7:1-10

2. Fujimura M, Myou S, Matsuda M, Amemiya T, Kamio Y, et al. (1998) Cough receptor sensitivity to capsaicin and tartaric acid in patients with Mycoplasma pneumonia. Lung 176: 281-288. 
3. Belvisi MG, Birrell MA, Khalid S, Wortley MA, Dockry R, et al. (2016) Neurophenotypes in Airway Diseases. Insights from Translational Cough Studies. Am J Respir Crit Care Med 193: 1364-1372.

4. Polverino M, Polverino F, Fasolino M, Andò F, Alfieri A, et al. (2012) Anatomy and neuro-pathophysiology of the cough reflex arc. Multidiscip Respir Med 7: 5.

5. Hegland KW, Bolser DC, Davenport PW (2012) Volitional control of reflex cough. J Appl Physiol (1985) 113: 39-46.

6. Dicpinigaitis PV (2003) Cough reflex sensitivity in cigarette smokers. Chest 123: 685-688.

7. Dicpinigaitis PV (2003) Short- and long-term reproducibility of capsaicin cough challenge testing. Pulm Pharmacol Ther 16: 61-65.

8. [No authors listed] (1995) Standardization of Spirometry, 1994 Update American Thoracic Society. Am J Respir Crit Care Med 152: 1107-1136.

9. Hankinson JL, Odencrantz JR, Fedan KB (1999) Spirometric reference values from a sample of the general U.S. population. Am J Respir Crit Care Med 159: 179-187.

10. Broeders ME, Molema J, Hop WC, Folgering HT (2005) Bronchial challenge, assessed with forced expiratory manoeuvres and airway impedance. Respir Med 99: 1046-1052.

11. Smith HJ, Reinhold P, MD. G (2005) Forced oscillation technique and impulse oscillometry. In: Gosselink R, H. S, eds. Lung Function Testing. Wakefield, UK: European Respiratory Society Journal Ltd 72-105

12. American Thoracic Society (1999) Recommendations for Standardized Procedures for the Online and Offline Measurement of Exhaled Lower Respiratory Nitric Oxide and Nasal Nitric Oxide in Adults and Children -1999. Am J Respir Crit Care Med 160:2104-2117.

13. Ternesten-Hasséus E, Bende M, Millqvist E (2002) Increased capsaicin cough sensitivity in patients with multiple chemical sensitivity. J Occup Environ Med 44: 1012-1017.

14. Haight RR, Gordon RL, Brooks SM (2006) The effects of age on exhaled breath nitric oxide levels. Lung 184: 113-119.

15. Dicpinigaitis PV, Alva RV (2005) Safety of capsaicin cough challenge testing. Chest 128: 196-202.

16. Chang AB, Phelan PD, Sawyer SM, Del Brocco S, Robertson CF (1997) Cough sensitivity in children with asthma, recurrent cough, and cystic fibrosis. Arch Dis Child 77: 331-334.

17. Dicpinigaitis PV, Grimm DR, Lesser M (1999) Cough reflex sensitivity in subjects with cervical spinal cord injury. Am J Respir Crit Care Med 159: 1660-1662.

18. Fujimura M, Ogawa $H$, Yasui $M$, Matsuda $T$ (2000) Eosinophilic tracheobronchitis and airway cough hypersensitivity in chronic nonproductive cough. Clin Exp Allergy 30: 41-47.

19. Ebihara S, Ebihara T, Kanezaki M, Gui P, Yamasaki M, et al. (2011) Aging deteriorated perception of urge-to-cough without changing cough reflex threshold to citric acid in female never-smokers. Cough 7:3.

20. Newnham DM, Hamilton SJ (1997) Sensitivity of the cough reflex in young and elderly subjects. Age Ageing 26: 185-188.

21. ClinicalTrials.gov (2012) Human Safety of Capsaicin Inhalation Challenge Testing for Young and Older Men (Capsaicin) at USF. In:
ClinicalTrials.gov, ed. web: US: Food and Drug Administration, National Institute of Health; After a research subject's death during an inhalation study using medications/drugs not approved for this route, the FDA prohibited human use of non-approved chemicals including capsaicin administered via inhalation.

22. Costanzo MT, Yost RA, Davenport PW (2014) Standardized method for solubility and storage of capsaicin-based solutions for cough induction. Cough 10: 6 .

23. Kopec SE, DeBellis RJ, Irwin RS (2002) Chemical analysis of freshly prepared and stored capsaicin solutions: implications for tussigenic challenges. Pulm Pharmacol Ther 15: 529-534.

24. Kopec SE, Irwin RS, Debellis RJ, Bohlke MB, Maher TJ (2008) The effects of Tween- 80 on the integrity of solutions of capsaicin: useful information for performing tussigenic challenges. Cough 4: 3 .

25. Vovk A, Bolser DC, Hey JA, Danzig M, Vickroy T, et al. (2007) Capsaicin exposure elicits complex airway defensive motor patterns in normal humans in a concentration-dependent manner. Pulm Pharmacol Ther 20:423-432.

26. Millqvist E (2000) Cough provocation with capsaicin is an objective way to test sensory hyperreactivity in patients with asthma-like symptoms. Allergy 55: 546-550.

27. Ternesten-Hasséus E, Larsson C, Larsson S, Millqvist E (2013) Capsaicin sensitivity in patients with chronic cough- results from a cross-sectional study. Cough 9: 5.

28. Ternesten-Hasséus E, Lowhagen O, Millqvist E (2007) Quality of life and capsaicin sensitivity in patients with airway symptoms induced by chemicals and scents: a longitudinal study. Environ Health Perspect 115: 425-429.

29. Gonda I (2006) Systemic delivery of drugs to humans via inhalation. J Aerosol Med 19: 47-53.

30. Vandenplas O, Malo JL (1997) Inhalation challenges with agents causing occupational asthma. Eur Respir J 10: 2612-2629.

31. Inoue K, Takano H, Yanagisawa R, Sakurai M, Ichinose T, et al. (2005) Effects of nano particles on antigen-related airway inflammation in mice. Respir Res 6: 106.

32. Maynard AD (2007) Nanotechnology: the next big thing, or much ado about nothing? Ann Occup Hyg 51: 1-12.

33. Maynard AD, Kuempel ED (2005) Airborne nonstructured particles and occupational health. Journal of Naoparticle Research 7:587-614.

34. Levine C, Faden R, Grady C, Hammerschmidt D, Eckenwiler L, et al. (2004) "Special scrutiny": a targeted form of research protocol review. Ann Intern Med 140: 220-223.

35. Kottow M (2004) The battering of informed consent. J Med Ethics 30: 565-569.

36. Vandenplas O, Suojalehto H, Aasen TB, Baur X, Burge PS, et al. (2014) Specific inhalation challenge in the diagnosis of occupational asthma: Consensus statement. Eur Respir J 43: 1573-1587. 